\title{
Ground-based detection of calcium and possibly scandium and hydrogen in the atmosphere of HD 209458b
}

\author{
N. Astudillo-Defru ${ }^{1,2}$ and P. Rojo ${ }^{1}$ \\ 1 Departamento de Astronomía, Universidad de Chile, Camino El Observatorio 1515, Las Condes, Santiago, Chile \\ e-mail: nicola.astudillo@obs.ujf-grenoble.fr \\ 2 UJF-Grenoble 1/CNRS-INSU, Institut de Planétologie et d'Astrophysique de Grenoble (IPAG) UMR 5274, 38041 Grenoble, France
}

Received 10 February 2012 / Accepted 17 June 2013

\begin{abstract}
Context. Since the first exoplanetary atmosphere detection using the Hubble Space Telescope, characterization of exoplanet atmospheres from the ground have been playing an increasingly important role in the analysis of these atmospheres thanks to the enhancement of telluric correction techniques. At present, several species have been discovered in the atmosphere of HD 209458b, all of them consistent with theoretical models.

Aims. Data acquired using the High Dispersion Spectrograph on the Subaru telescope are re-analysed. We expect to discover new species in the atmosphere of the exoplanet HD 209458b. In addition to shedding light on the atmospheric composition, we will derive the radial extension of the absorbents present in the atmosphere of the exoplanet.

Methods. We present an alternative method for correcting the telluric effects through the analysis of variations in spectral lines with the airmass. To search for absorptions due to an exoplanetary atmosphere we implemented an algorithm to automatically search for all the features presenting an atmospheric signature in the transmission spectrum and through the wavelength range in the data. To estimate uncertainties we performed a bootstrapping analysis.

Results. Absorption excess due to the transitions of calcium at $6162.17 \AA$ and $6493.78 \AA$, scandium at $5526.79 \AA$, hydrogen at $6562.8 \AA$, and the sodium doublet are detected in the transmission spectrum at a level of $-0.079 \pm 0.012 \%,-0.138 \pm 0.013 \%$, $-0.059 \pm 0.012 \%,-0.123 \pm 0.012 \%,-0.071 \pm 0.016 \%$ using pass-bands of $0.5 \AA, 0.4 \AA, 0.5 \AA, 1.1 \AA$, and $0.6 \AA$, respectively. Conclusions. Models predict strong absorption in the sodium resonance doublet which was previously detected, also in this analysis. However, this is the first report of calcium and possibly scandium in HD 209458b, including the possible ground-based detection of hydrogen. Calcium is expected to condense out in the atmosphere of this exoplanet; therefore, confirmation of these results will certainly imply a review of theoretical models.
\end{abstract}

Key words. techniques: spectroscopic - planets and satellites: atmospheres - planets and satellites: composition - atmospheric effects

\section{Introduction}

Since the first detection of an extrasolar planet (Mayor \& Queloz 1995) the radial-velocity and transit method have allowed the measurement of dynamics and bulk properties of hundreds of exoplanets. The first method is responsible of the majority of known planets and give us the minimum mass $m \sin (i)$, among other parameters. The second identified one third of the known planets, added thousands of candidates, and proved planet radii and inclinations. Both methods are complementary, letting us to know the planet true mass. Moreover, transits present a unique opportunity to characterize exoplanets. Transmission spectroscopy allows us to measure the absorption of radiation passing through the optically thin exoplanet atmosphere and to decode the composition and physical parameters of the outer atmosphere (Charbonneau et al. 2002, hereafter C2002).

Absorption from exoplanetary atmospheres are expected to be of the order of $10^{-3}$ for a hot-Jupiter planet orbiting a Sun-like star. Therefore high signal-to-noise ratio $(\mathrm{S} / \mathrm{N})$ data are needed to measure this absorption. Regarding photon collecting capacity, ground-based telescopes have an advantage over space-based. However, data acquired using ground-based instruments must be corrected to deal with variations introduced by the Earth's atmosphere. In previous work, these corrections are mainly done by observation of a reference star (simultaneously or not) or the creation of a synthetic telluric spectrum (Bean et al. 2010; Redfield et al. 2008; Langland-Shula et al. 2009, hereafter R2008 and L2009, respectively).

Traditionally, theoretical models have been used to predict which transitions are prominent enough to be easily distinguishable from noise (Burrows et al. 2010; Fortney et al. 2010). Detected components in the transiting HD 209458b atmosphere agree with these models (C2002, Vidal-Madjar et al. 2003, 2004; Beaulieu et al. 2010; Désert et al. 2008). We note that previous detections were reported in a single spectral line (or doublet) or photometric detection instead of a suite of transitions of a particular species. Although much work has been done to date, more studies need to be conducted to confirm some of the previous results and observation-models agreements, as well as to improve techniques for precise constraining of abundances.

The purpose of present study was to perform a so-called blind search in the whole transmission spectrum of HD 209458b to automatically detect strong and missed absorption produced in the exoplanetary atmosphere for every strong transition reported in databases. This research propose an alternative to the problem of correcting for non-desired telluric effects on transmission 

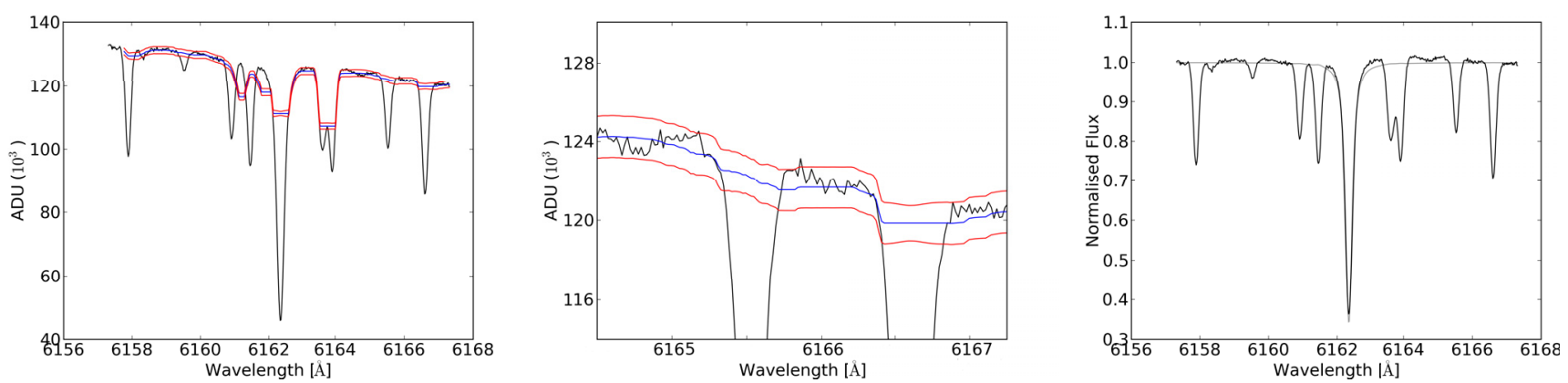

Fig. 1. Local normalisation around a spectral line. Left: a median filtered array (middle blue line), the filter width was chosen to be 1.25 times the full width at half maximum (FWHM), typically FWHM $\sim 0.4 \AA$, which corresponds to 50 pixels given the instrument resolution. Pixels within $3 \times F W H M$ and with intensity beyond 3 Poisson-statistics sigmas from the median filter (red curves) are discarded. Middle: zoom to the median filter. The accepted pixels were used to fit a third order polynomial. Right: the normalized spectrum after division by the polynomial and the Voigtian fit (grey) to the stellar line.

spectroscopy. The topic is especially important in the search for biomarkers in exoplanetary atmospheres.

\section{Observations, data reduction, and analysis}

The science archive SMOKA (Baba et al. 2002) was used to obtain data collected with the High Dispersion Spectrograph (HDS) on the Subaru telescope. These HD 209458 data have been described in Winn et al. (2004); Narita et al. (2005); Snellen et al. (2008), hereafter W2004, N2005, and S2008, respectively. The entrance slit was $0.8^{\prime \prime}$ and the resolution was $R \sim 45000$.

After applying the $\mathrm{S} 2008$ correction $^{1}$ for the CCD nonlinearity effects, we followed the HDS IRAF Reduction Manual available on the NAOJ website ${ }^{2}$. We used 29 spectra frames each with a total exposure time of 500s. The resulting $\mathrm{S} / \mathrm{N}$ varies between 300 and 450 per pixel in the continuum. We concentrated on the red CCD that contains 21 orders covering from $5500 \AA$ to $6800 \AA$.

The Knutson et al. (2007) ephemeris was used to select the in-transit and out-of-transit frames. We independently and automatically analysed the region around each of the transitions indexed in The Interactive Database of Spectral Standard Star Atlases $^{3}$ (Lobel 2008). This database lists the line parameters, including rest wavelength, oscillator strength $\left(g_{i} f_{i j}\right)$, expected line depth, and responsible element for telluric and stellar transitions for a subset of template standard stars. For HD 209458 $(\mathrm{G} 0 \mathrm{~V})$ we used the solar spectrum template because it is the closest spectral type. Using this database limited our study to transitions present in the host star, i.e. we were restricted to exoplanet elements that are also present in the parent star atmosphere.

\subsection{Data preparation}

A Doppler correction for each Echelle order was applied to locate observable transitions from the database. We used four to eight transitions to obtain the linear dependence between the rest and observed wavelength difference as a function of the observed wavelength. This procedure was less accurate than calculating the radial velocities by the correlation-cross-function but precise enough to find the Doppler-shifted transitions from the database.

\footnotetext{
$1 F_{\text {cor }}=F_{\text {raw }}-3.0 \times 10^{-6} \times F_{\text {raw }}^{2}$.

2 http://www.naoj.org/Observing/Instruments/HDS/

3 http://spectra.freeshell.org/spectroweb.html
}

We did not follow the method used in W2004, N2005, and S2008 to correct the variations in the systematic blaze function. Instead, we normalized locally ${ }^{4}$ around each analysed transition by implementing a median filter, sigma clipping, and using a third order polynomial (details in Fig. 1). Then, we applied the following automated selection criteria to each spectral line to identify those with the best conditions for the further analysis.

- Since faint transitions are more difficult to detect, we selected those stellar lines having a minimum line depth of $10 \%$ in the database (i.e. at the solar spectrum).

- Zones with bad automatic normalisation were discarded by obtaining pixel by pixel the residuals from the normalized continuum and its median. These values were sorted. Selected transitions have $70 \%$ of sorted residuals below the empirical value of 0.009 .

- To discard blended lines we calculated the differences in wavelength and normalized flux between the pixel with the lowest value (line core) and the centre of a Voigtian fit. The differences for selected lines were less than the empirical value of $0.06 \AA$ ( $~ 3$ pixels) in wavelength and less than 0.06 in normalized flux.

- Stronger transitions are easier to detect in the transmission spectrum. Therefore, we used the oscillator strength as a proxy. We selected those transition having an oscillator strength value greater than the value of $\mathrm{Na} \mathrm{D}\left(\log \left(g_{i} f_{i j}\right)=\right.$ -0.184 ) which was detected in HD 209458b using this data set (S2008). The transitions strength of $\mathrm{Ca}, \mathrm{Fe}, \mathrm{Mg}, \mathrm{Mn}, \mathrm{Na}$, and Sc empirically correlated with oscillator strength.

Finally, we note that most of the lines present an "s" shape in the transmission spectrum. Jensen et al. (2012) report the same "s" shape for $\mathrm{H} \alpha$ and give some possible interpretations. However, in our analysis we found that this unusual feature is not planetary phase-correlated because it also appears when comparing only out-of-transit data. To correct these relative shifts between spectra we calculated the centre of each stellar line by the Voigtian fit. This centre was used to obtain the difference in wavelength between the absorption in a reference exposure and the rest of frames. Then, we aligned the spectral line for each exposure to a common centre using a cubic spline re-sampling. Figure 2 summarizes the selection criteria by examples.

\footnotetext{
$45 \AA$ and $7 \AA$ around each transition, equivalent to 250 and 350 times the spectral resolution, respectively.
} 

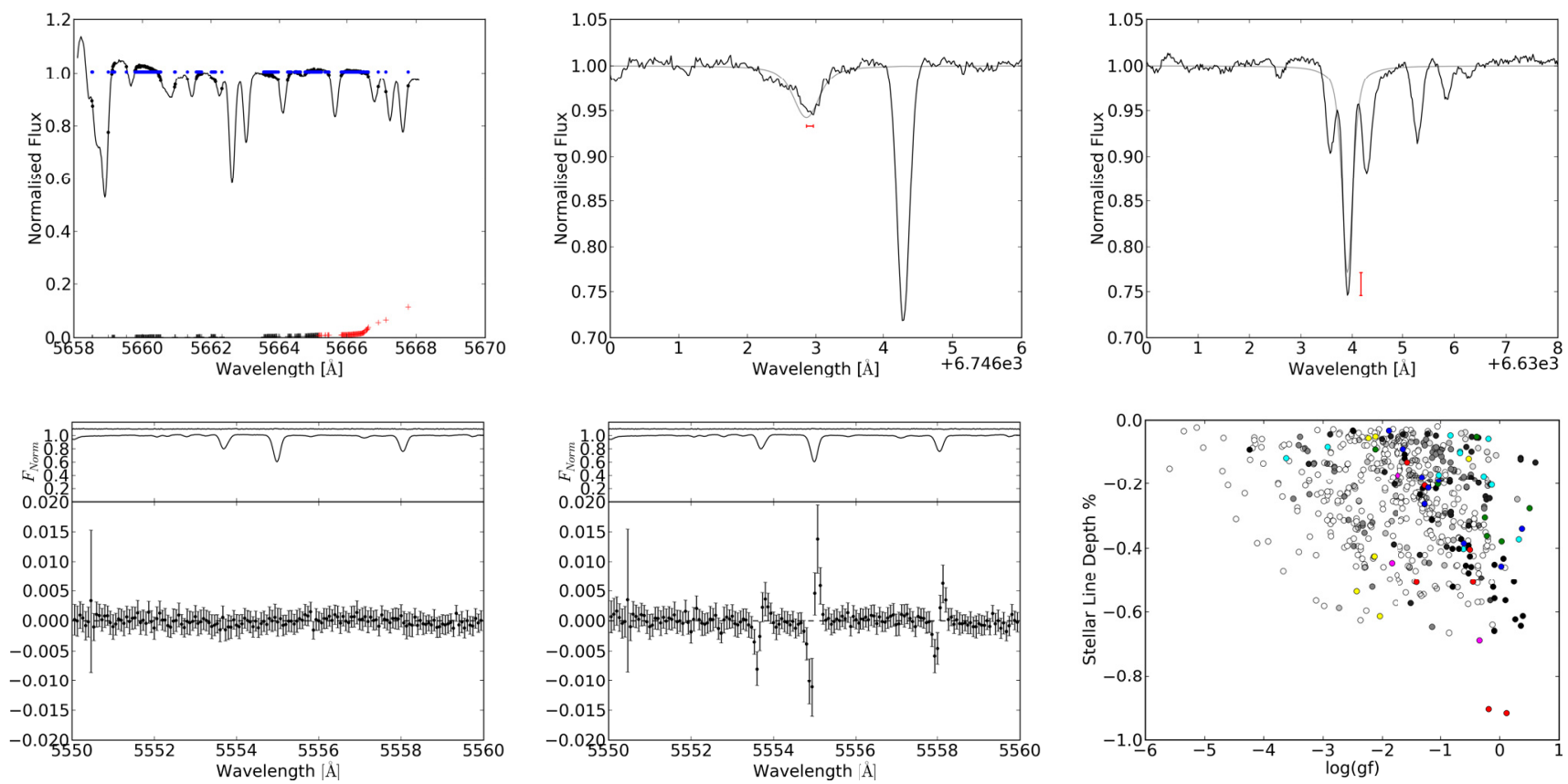

Fig. 2. Top row: cases of transitions rejected by the selection criteria. From left to right: a crowded zone: black and blue points are pixels considered as continuum by the median filter and the median value, respectively. The sorted residuals are also plotted, where the 70\% limit (black to red) is over 0.009. An asymmetrical blend: the red segments represents the wavelength difference. A symmetrical blend: the red segment shows the flux difference. Bottom row: the transmission spectrum without (left) and with (middle) the local shift correction. The stellar spectrum is shown at the top. Right: stellar line depths are generally stronger with greater oscillator strength values. Several species are plotted with different colours.

\subsection{Telluric corrections}

Spectra recorded using ground-based instruments suffer from the imprints of the Earth's atmosphere which can vary in time for different reasons. Therefore, those data must be corrected from telluric features producing spurious signals in the transmission spectrum. A powerful method for recognising telluric lines was implemented using the airmass in the radiative transfer equation. The solution of this equation assuming only absorption is $\ln \left(I / I_{0}\right)=N k_{\lambda} s$, where $I$ is the intensity at certain wavelength $\lambda$, $I_{0}$ is the source intensity, $N k_{\lambda}$ is the optical depth at zenith, and $s$ is the airmass. We ignore slit-losses as first trial.

We derived $N k_{\lambda}$ and its error through a linear regression to $\ln (I)$ as a function of the airmass $s$ at a mid-exposure. This procedure was done only using out-of-transit spectra because while the transit starts, a relatively abrupt variation in $F_{\lambda}$ is expected if a detectable element is present in the exoplanetary atmosphere blocking photons at $\lambda$. This method yields a telluric spectrum (see Fig. 3, top) which is clearly consistent with the telluric spectrum shown in Fig. 1 from L2009 or S2008. Any slit-losses were thus empirically shown of second order.

Each spectrum was divided by $\mathrm{e}^{N k_{\lambda}\left(s_{i}-s_{\mathrm{Ref}}\right)}$, where $s_{\text {Ref }}$ is the average airmass of in-transit spectra. We note that some strong tellurics are not fully corrected as features appear in telluric line positions in the transmission spectrum. We attribute this to short timescale variations in telluric species content or second-order deviations from the linear dependence $\ln (I) \propto s$, like slit-losses. We paid special attention when a feature was present in both the transmission and the telluric spectrum, like in $\mathrm{Na} \mathrm{D}$ vicinity.

\subsection{Features in the transmission spectrum}

For each transition, we created a central pass-band $(\mathrm{C})$ centred in the interest line and two adjacent control pass-bands, blue (B)

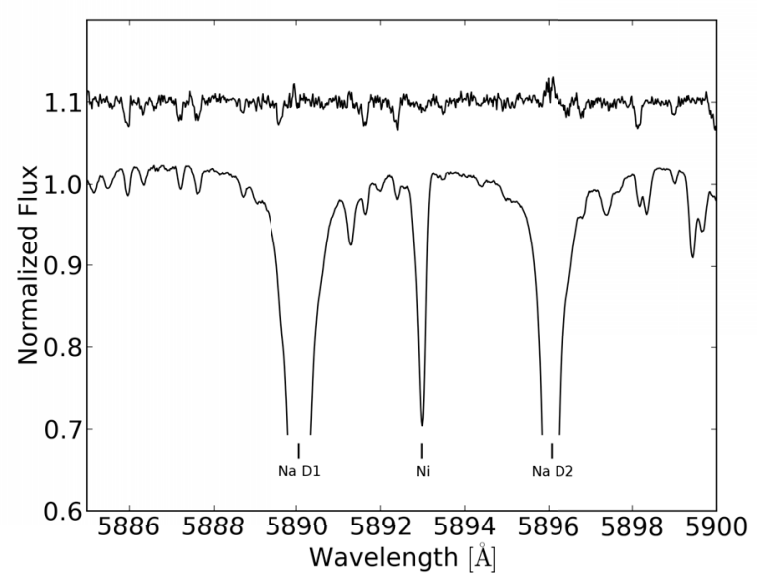

Fig. 3. Example of telluric absorptions in HDS spectra around the Na I D. HD 209458 normalised spectrum is shown while the telluric spectrum is shown above it with an offset of 0.1 .

and red (R). From R2008, and Jensen et al. (2011, 2012) we note that features in the transmission spectrum fall within stellar line profiles because even if spectral lines are broader in atmospheres such as HD 209458b the strongest absorption occurs at the core. This suggested that we should select pass-band sizes proportional to the stellar line width. Pass-band sizes were given by $\times 3, \times 4.5$, and $\times 6$ the width of the Voigtian fit to each stellar transition. We note that originally C2002 used three central band-passes because they did not know the precise width of the sought feature.

To automatically detect features in the transmission spectrum we calculated the relative flux based both with R2008 and S2008 methods. The depth of the light curve should be consistent in both cases. 


\subsubsection{R2008 based analysis}

This analysis visualises the transmission spectrum along wavelength. In-transit $\mathcal{F}_{\text {In }}$ and out-of-transit $\mathcal{F}_{\text {Out }}$ spectra were stacked separately reaching a $S N R \sim 1600$ and $S N R \sim 1200$, respectively; $\mathcal{F}_{\text {In }}(\lambda)$ and $\mathcal{F}_{\text {Out }}(\lambda)$ errors were obtained with the standard deviation of normalized flux by pixel across in-transit and out-of-transit frames. The relative flux $F_{\text {Rel }}(\lambda)=\overline{\mathcal{F}_{\text {In }}} / \overline{\mathcal{F}_{\text {Out }}}$ was computed and then the transmitted signal $F_{\text {Trans }}$ was calculated for each pass-band as follows

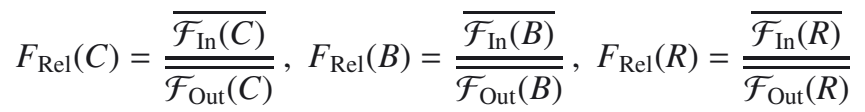

$F_{\text {Trans }}=F_{\text {Rel }}(C)-\left[F_{\operatorname{Rel}}(B)+F_{\operatorname{Rel}}(R)\right] / 2$.

To deal with systematic and random errors a statistical diagnostic of the stability of a measured absorption was done by bootstrapping analysis.

Three different scenarios were created following R2008's Sect. 3.2. (1) An out-out comparison where a randomly selected subset of out-of-transit data (7 frames) were used as in-transit data and the non-selected out-of-transit (4 frames) were used as out-of-transit exposures; each data subset was then used to calculate the transmitted signal. Since there should be no difference among the transmitted signal for these populations, the resulting distribution is expected to be centred at zero. (2) Similarly, an in-in comparison where a randomly selected subset of in-transit exposures (11 frames) were compared to the rest of in-transit data. The resulting distribution is also expected to be centred at zero. (3) An in-out comparison where an increasing number of spectra (up to half of the sample) were removed from the intransit sample and the remaining were compared with the outof-transit data set. The resulting distribution is expected to be centred at the measured value of the transmitted signal using the all data set.

Three thousands iterations were selected because the width of distributions in the in-in, out-out and in-out scenarios do not increase significantly over this number. We considered $1 \sigma$ of the in-out scenario as the uncertainties for our later results.

\subsubsection{S2008-based analysis}

This analysis visualises the light curve derived from the transmission spectrum in a specific wavelength. We perform the spectrophotometry deriving $F_{\mathrm{Rel}}(t)$ for each exposure using the same pass-bands described in Sect. 2.3. Derived light curves were normalized and the transmitted signal $F_{\text {Trans }}$ (the light curve depth) was obtained by

$F_{\text {Rel }}(t)=\frac{2 \overline{F_{\mathrm{C}}}}{\overline{F_{\mathrm{B}}}+\overline{F_{\mathrm{R}}}}, F_{\text {Trans }}=\overline{F_{\text {Rel }}\left(t_{\text {In }}\right)}-\overline{F_{\text {Rel }}\left(t_{\text {Out }}\right)}$,

where the uncertainty of the relative flux $\sigma_{F_{\mathrm{Rel}}(t)}$ was estimated by considering only photon noise.

Unlike S2008, however, we do not fit a model with stellar limb-darkening. Instead, we measure the absorption as the difference between the in-transit and out-of-transit averages. Stellar limb darkening is not expected to significantly affect the transmitted signal since we used narrow band-passes and spectra were normalized by the local continuum. As demonstrated by R2008 using stellar models, and depending on the spectral line, the error for not considering limb-darkening accounts to a negligible $\lesssim 10^{-5}$. On the other hand, using the wrong limb-darkening law can be misleading (see Sect. 3.1).
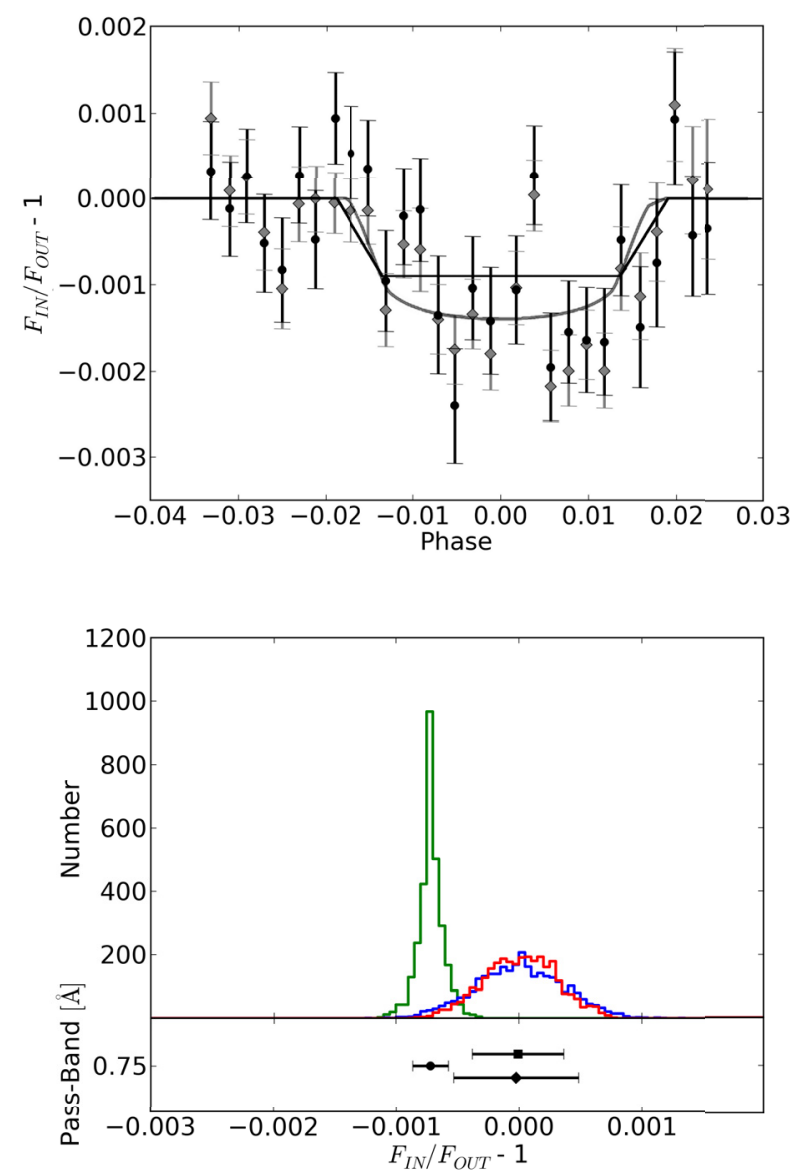

Fig. 4. Comparison from $\mathrm{Na} I \mathrm{D}$ with previous detection reported in this data set where the used pass-band was $0.75 \AA$. Top panel: transit photometry derived by S2008 (grey) and this work (black). The correction proposed by S2008 for CCD non-linearity was applied in this work. Solid lines represent the transit fits for this work (black) and S2008 (grey). The latter used a scaled version of HST photometry integrated over 582 and $638 \mathrm{~nm}$ which includes limb darkening contribution. Bottom panel: R2008 based analysis for the Na I D transmitted signal and its uncertainty derived from the bootstrap analysis. Green colour represent the in-out scenario, blue colour in-in scenario, and red colour the out-out scenario. The centre and $1 \sigma$ of each distribution is shown at the bottom.

\section{Results and analysis}

Previous sodium and hydrogen detections were confirmed and new calcium and possibly scandium features resulted in significant absorption in the transmission spectrum from 29 transitions satisfying our selection criteria described in Sect. 2.1 and over 2200 transitions automatically analysed in the twenty-nine spectra.

\section{1. $\mathrm{NaI}$}

As is expected from the previously analysed data set (S2008), absorption excess in $\mathrm{Na} I \mathrm{D}$ was measured at a level of $-0.071 \pm$ $0.016 \%$ ( $\times 3$ band $),-0.043 \pm 0.012 \%(\times 4.5$ band $)$, and $-0.045 \pm$ $0.010 \%$ ( $\times 6$ band). The averaged Voigtian width in Na D transitions was $0.21 \AA$. We note that the effect of stellar limb darkening is an order of magnitude less than our obtained statistical error.

As comparison, C2002, S2008, and L2009 measured an absorption of $-0.023 \pm 0.006 \%,-0.135 \pm 0.017 \%$, and $-0.109 \pm$ $0.026 \%$ in the sodium doublet using their own bandwidths, 
N. Astudillo-Defru and P. Rojo: Ground-based detection of Ca I and possibly Sc II and H I in the atmosphere of HD 209458b
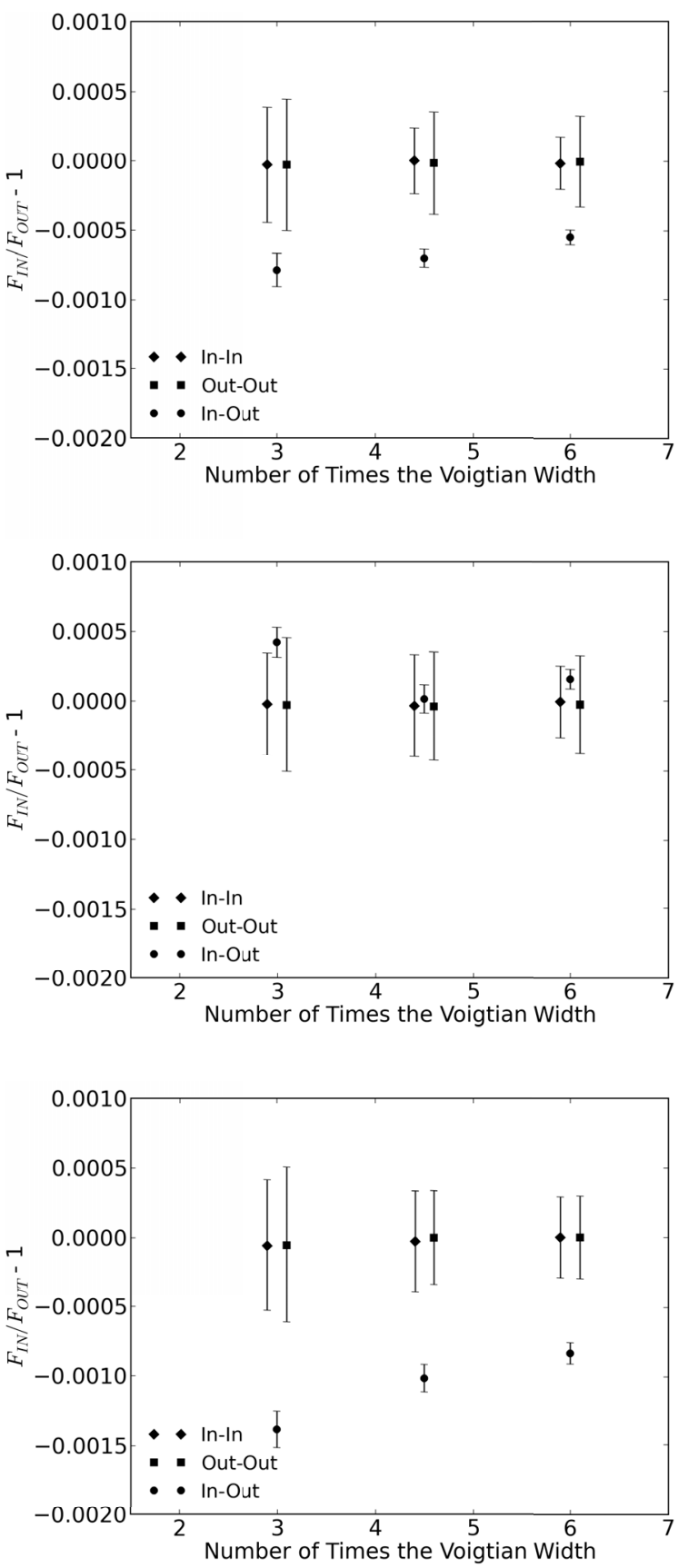

Fig. 5. Transmitted signals of the Ca I transitions at $6162.27 \AA$ (top panel), $6439.1 \AA$ (middle panel), and $6493.78 \AA$ (bottom panel) using pass-bands of $\times 3, \times 4.5$, and $\times 6$ the stellar line width derived from fitting a Voigt profile. The significance of measurements were derived from the in-out scenario in the bootstrap analysis described in Sect. 2.3.1. The noise levels were derived from the in-in and out-out scenarios of the bootstrap analysis.

respectively. Selecting the same pass-bands used in S2008, our detection level was $-0.072 \pm 0.015 \%(0.75 \AA)$. The top panel in Fig. 4 shows the light curve derived for Na I D absorption excess using the $0.75 \AA$ pass-band, while the bottom panel shows the associated bootstrap distributions (top) where we specify its central values and $1 \sigma$ uncertainties (bottom). We note that almost all our light curve points are within the $\mathbf{S} 20081 \sigma$ values. In fact, using our same transit-fitting modelling on the S2008 data (average-in minus average-out) we obtain a difference between their depth and ours of $0.016 \%$, which is in agreement

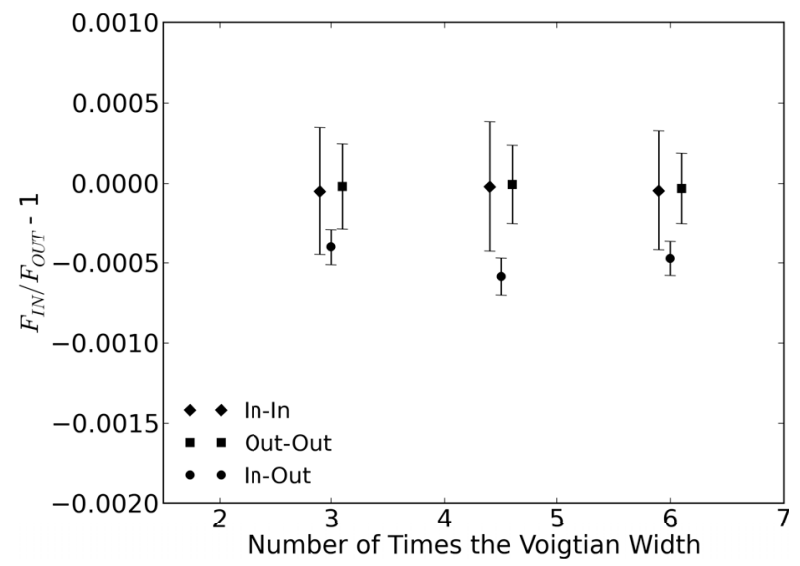

Fig. 6. Same as Fig. 5 but for Sc II at $5526.79 \AA$.

with the errors. The disagreement with their published $\mathrm{Na} \mathrm{D}$ absorption $(-0.135 \pm 0.017 \%)$ therefore seems to come primarily from their choice of fitting a model with a limb darkening law adapted from a broadband observation.

\section{2. $\mathrm{CaI}$}

Eight-five $\mathrm{Ca}$ I transitions were analysed and transitions at 6162.17, 6439.08 and 6493.78 A passed all four selection criteria. Transmitted signals for each Ca I transition and selected pass-bands are shown in Fig. 5.

The measurements for Ca I, which at $6162.27 \AA$ has an absorption excess detected at $10 \sigma$, are $-0.079 \pm 0.012 \%(\times 3$ band $)$, $-0.070 \pm 0.007 \%$ ( $\times 4.5$ band $)$, and $-0.055 \pm 0.005 \%$ ( $\times 6$ band $)$. The Voigtian fit to the stellar line gives a width of $0.17 \AA$. Measurements through the different pass-band sizes at $6439.1 \AA$ are within $1 \sigma$ error derived from the in-in and out-out scenarios of the bootstrap analysis and therefore are not considered as a detectable signal. These values are $0.042 \pm 0.010 \%$, $0.001 \pm 0.010 \%$, and $0.016 \pm 0.007 \%$ in the $\times 3, \times 4.5$, and $\times 6$ bands, respectively. The Voigtian width used for the passband sizes was $0.15 \AA$. Finally, the Ca I transition at $6493.78 \AA$ present a strong absorption excess at a level of $-0.138 \pm 0.013 \%$ ( $\times 3$ band $),-0.102 \pm 0.010 \%$ ( $\times 4.5$ band $)$, and $-0.083 \pm 0.008 \%$ ( $\times 6$ band), where the stellar Voigtian width was $0.14 \AA$.

We derived a radial extent ${ }^{5}$ of $2537 \pm 391 \mathrm{~km}(4.6 \times \mathrm{H})$, $2267 \pm 212 \mathrm{~km}(4.1 \times \mathrm{H})$, and $1770 \pm 171 \mathrm{~km}(3.2 \times \mathrm{H})$ for $\mathrm{Ca} \mathrm{I}$ at $6162.27 \AA ; 4419 \pm 429 \mathrm{~km}(8.0 \times \mathrm{H}), 3271 \pm 325 \mathrm{~km}(5.9 \times \mathrm{H})$, and $2676 \pm 252 \mathrm{~km}(4.9 \times \mathrm{H})$ for $\mathrm{Ca}$ I at $6493.78 \AA$ in $\times 3, \times 4.5$, and $\times 6$ pass-bands, respectively, where $H$ is the scale height and we used $R_{\mathrm{p}}=1.38 R_{\mathrm{Jup}}{ }^{6}$ and $R_{\star} / R_{\mathrm{p}}=0.11384 / 0.01389$ (Southworth 2010).

Transitions at $6162.27 \AA$ and $6493.78 \AA$ show the expected rise of absorption toward smaller pass-bands. Surprisingly, Ca I at $6439.1 \AA$ presents no significant signal. However, we believe that this could be due to some extra systematics from that particular region of the detector, which is near the CCD edge and receives about $20 \%$ less photons $(S N R \sim 250$ per pixel at the middle of the line profile). This is further supported by the fact that not even a hint of the tellurics in the region are detected even though they have a $\log \left(g_{i} f_{i j}\right)$ as strong as that of tellurics positively detected in different regions.

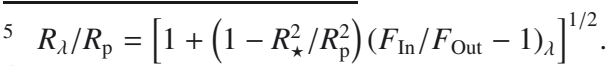

$6 \quad R_{\text {Jup }}=71492[\mathrm{~km}]$.
} 

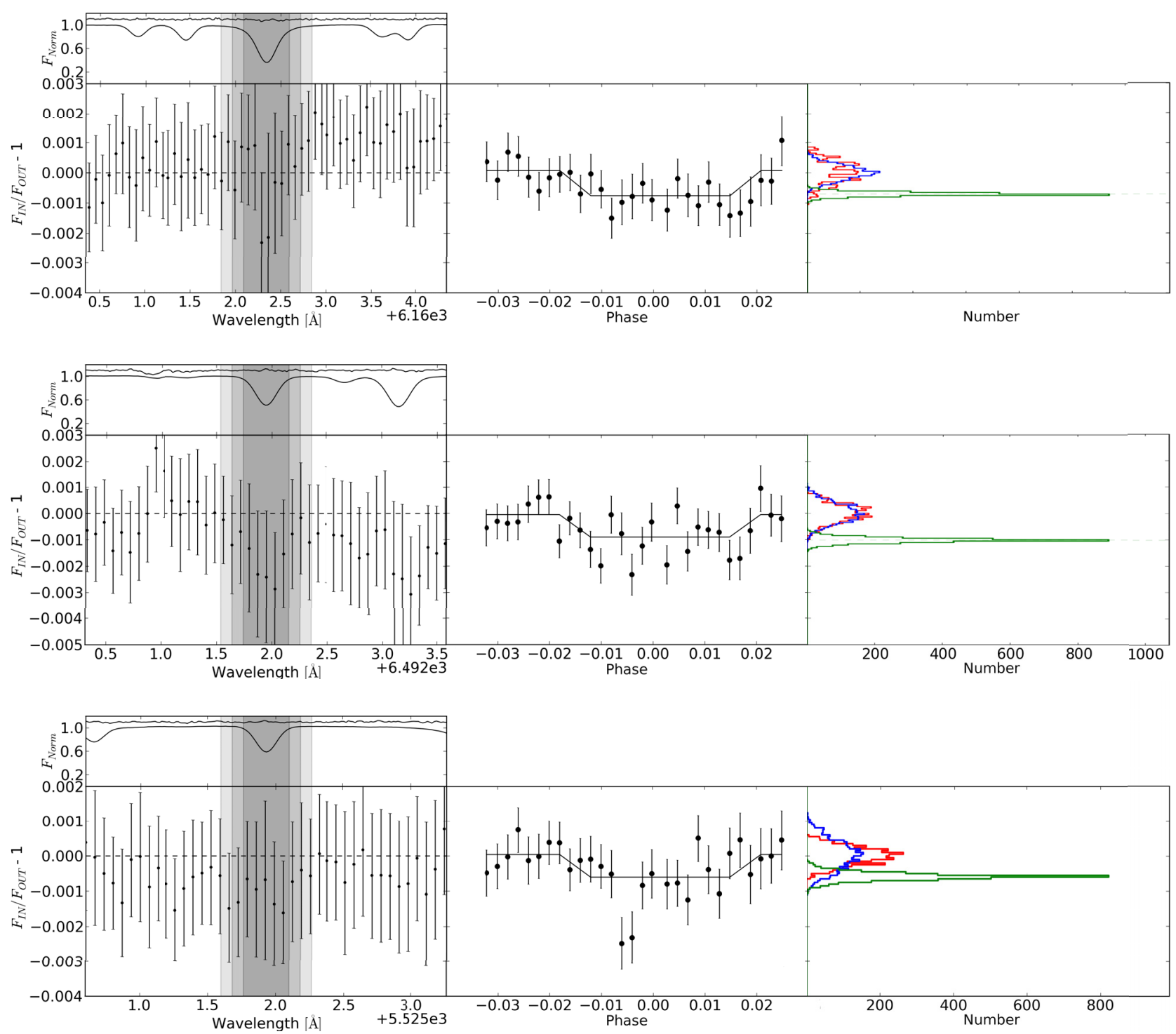

Fig. 7. Analysis of the transitions of Ca I at $6162.27 \AA$, Ca I at $6493.8 \AA$. and Sc II at $5526.79 \AA$ are shown in the first, second, and third row, respectively. Left panels: absorption excess in the binned transmission spectrum. For reference, the normalised stellar spectrum and the telluric spectrum ( 0.1 offset and 3 times magnified) are shown at the top of each panel. The shaded zones represent the pass-bands sizes. Middle panels: light curves derived from spectrophotometry using the $\times 4.5$ band for both $\mathrm{Ca}$ I transitions and for Sc II. Flat light curves represent the averages of in-transit and out-of-transit data. Right panels: derived bootstrap distributions showing the detection stability: blue represents the in-in distribution, red the out-out distribution, and green is the in-out distribution.

\subsection{Sc II}

Thirty-six Sc transitions are present in the considered optical range, but only the Sc II transition at $5526.79 \AA$ A passed our selection criteria. Figure 6 shows results computed for each selected pass-band and their significance from the bootstrapping.

The detection is at $>4 \sigma$, at a level of $-0.059 \pm 0.012 \%$ and $-0.047 \pm 0.011 \%$ for the $\times 4.5$ and $\times 6$ pass-bands, respectively. The Voigtian width of the stellar line used in the pass-band sizes is $0.11 \AA$. However, for the $\times 3$ band the measured value is within $1 \sigma$ error derived from the in-in and out-out scenarios of the bootstrap analysis. Hence, this $-0.040 \pm 0.012 \%$ measurement cannot be considered a detectable signal. As no telluric transition is reported in the used database, possible explanations are systematic errors or the presence of this elements in the exoplanetary atmosphere producing an unexpected emission, which may produce a fainting effect in the rise of absorption toward smaller pass-bands.

Nevertheless, the light curve in the $\times 4.5$ pass-band clearly shows the transit shape and is supported by the bootstrap analysis. Assuming that the absorption comes from the exoplanetary atmosphere and using HD 209458 physical parameters, we obtained a radial extent of $1896 \pm 379 \mathrm{~km}(3.4 \times \mathrm{H})$ and $1532 \pm 349 \mathrm{~km}(2.8 \times \mathrm{H})$ using the $\times 4.5$ and $\times 6$ pass-bands.

\section{4. $\mathrm{H} \alpha$}

Recently Jensen et al. (2012) report $\mathrm{H} \alpha$ detection in the atmosphere of HD 189733b. They analysed others exoplanets, including HD 209458b. Our algorithm did not automatically analyse this hydrogen transition because we used a narrower $5 \AA$ and $7 \AA$ window to normalise locally. And so this transition was rejected 
N. Astudillo-Defru and P. Rojo: Ground-based detection of Ca I and possibly Sc II and H I in the atmosphere of HD 209458b
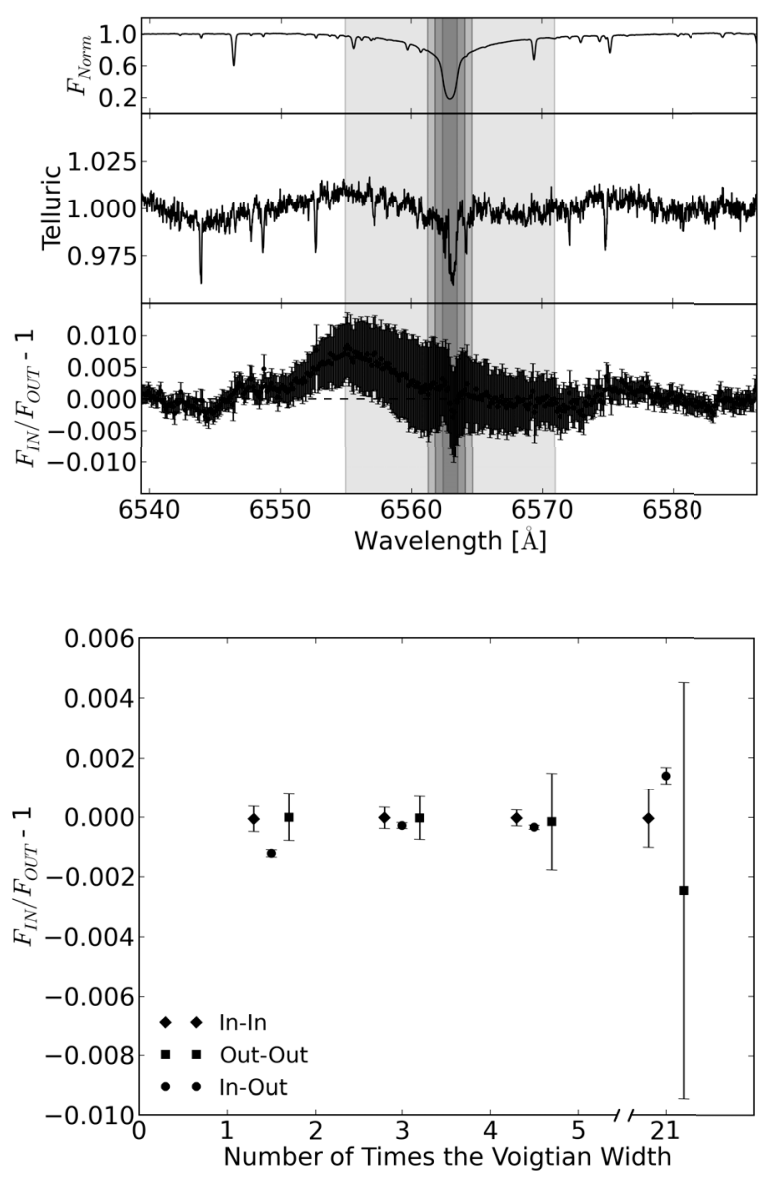

Fig. 8. Top panel: from top to bottom, HD 209458 spectrum near $\mathrm{H} \alpha$ transition, our derived telluric spectrum, and the transmission spectrum. The selected pass-bands are $\times 1.5, \times 3, \times 4.5$ the stellar line width, and the $16 \AA$ A pass-band used in Jensen et al. (2012). Bottom panel: transmitted signal measured in each used spectral window, including the $\times 21$ (16 $\AA$ ). This panel shows a possible detection of absorption excess due $\mathrm{H} \alpha$ in the narrower pass-band.

by our selection criteria. Changing the window size to $25 \AA$, using an estimated FWHM of $1.5 \AA$ for the $\mathrm{H} \alpha$ transition and rejecting pixels within 10 times the FWHM (see Sect. 2.1) the normalisation is satisfying.

We used pass-bands sizes of $\times 1.5, \times 3$, and $\times 4.5$ the width of the stellar line, where the Voigtian fit gives a stellar width of $0.75 \AA$. We also selected the $16 \AA$ spectral window used in Jensen et al. (2012). Measurements through these pass-bands results in $-0.123 \pm 0.012 \%,-0.029 \pm 0.010 \%,-0.035 \pm 0.008 \%$, and $0.140 \pm 0.027 \%$ in the $\times 1.5, \times 3, \times 4.5$, and $16 \AA$ pass-bands, respectively.

The top panel of Fig. 8 shows only one significant signal detected in the narrower pass-band. However, this signal needs careful interpretation because, even if the transmission spectrum (Fig. 8, bottom panel) shows an unusual feature in the bluer wing and an absorption near the core of stellar $\mathrm{H} \alpha$, this absorption match with the telluric spectrum and therefore incorrect telluric variations at water vapour transition locations may produce a spurious signal.

For HD 209458b, our signal detected in the $\times 1.5$ $(1.125 \AA)$ band is equivalent to a radial extent of $3928 \pm 397 \mathrm{~km}$ $(7.1 \times \mathrm{H})$. For the $L y \alpha$ transition Vidal-Madjar et al. (2003) obtained $15 \pm 4 \%$ absorption using a central pass-band between $1215.15 \AA$ and $1216.1 \AA$. In comparison, for HD 189733b

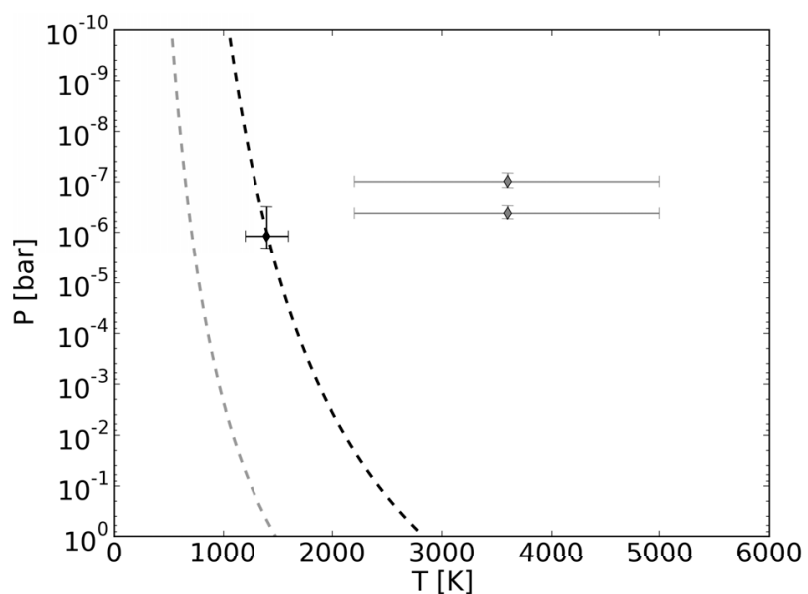

Fig. 9. Vapour pressure curves are represented by dashed lines. The grey dashed line represents the calcium vapour pressure curve and the black dashed line represents the scandium vapour pressure curve. Grey and black dashed diamonds show the temperature and pressure conditions from the measured altitudes for calcium and candium, respectively.

Jensen et al. (2012) report an $\mathrm{H} \alpha$ detection at a level of $-0.0872 \pm 0.0148 \%$ using a $16 \AA$ pass-band.

\subsection{On the $\mathrm{Ca}$ I and Sc II detection feasibility}

The Clausius-Clapeyron equation ${ }^{7}$ may be useful to analyse the phase diagram of a chemical element through the temperature and pressure. Altitudes $\left(z_{\mathrm{Sc}, \mathrm{Ca}}\right)$ derived previously for the absorbents in the atmosphere were used to obtain the atmospheric pressure-temperature conditions for HD 209458b (Vidal-Madjar et al. $2011 \mathrm{~b}$,a), where we considered isothermal layers ${ }^{8}$.

The physical parameters of calcium and scandium were obtained from Lide, David R. (2010). Figure 9 shows the derived vapour pressure curves and pressure-temperature conditions derived from altitudes $(1896 \pm 379 \mathrm{~km}$ for Sc II, and $2537 \pm 396 \mathrm{~km}$ and $4419 \pm 429 \mathrm{~km}$ for Ca I at $6162.27 \AA$ and $6493.78 \AA$, respectively). We note that calcium clearly remains in the gaseous phase while scandium grazes the vapour pressure curve in the liquid state; Sc clouds could thus be present.

Models of the atmosphere have predicted condensation of $\mathrm{Ca}$, but this condensation via $\mathrm{CaTiO}_{3}$ does not seem to necessarily imply the vanishment of $\mathrm{Ca}$, as $\mathrm{O}$ and possibly $\mathrm{TiO}$ have been previously detected in the atmosphere of HD 209458b (Vidal-Madjar et al. 2004; Désert et al. 2008).

\section{Conclusions}

We present the first ground-based detection of calcium and possibly scandium and hydrogen in the transmission spectrum of HD 209458b: Ca I at 6162.17 $\AA$ and $6493.78 \AA$, Sc II transition at $5526.79 \AA$, and $\mathrm{H} \alpha$ presents an absorption excess of $(-7.9 \pm 1.2) \times 10^{-4},(-13.8 \pm 1.3) \times 10^{-4},(-5.9 \pm 1.2) \times 10^{-4}$, and $(-12.3 \pm 1.2) \times 10^{-4}$ in the $\times 3, \times 3, \times 4.5$, and $\times 1.5$ pass-band, respectively. The transitions $\mathrm{Na} \mathrm{D}$ and $\mathrm{Ca}$ I show the expected rise of observed altitudes toward smaller pass-bands, while Sc

$7 \quad P_{\text {Vap }}=P_{\text {Vap }, 0} \exp \left(-\frac{\Delta H}{R}\left[\frac{1}{T}-\frac{1}{T_{0}}\right]\right)$, where $P_{\text {Vap }}$ is the vapour pressure, $\Delta H$ is the latent heat of the phase transition, $R$ is the specific gas constant, and $T$ the temperature.

${ }^{8} P=P_{0} \exp \left(-\frac{z_{\mathrm{Sc}, \mathrm{Ca}}-z_{\min }}{H}\right)$, where $P$ is the pressure and $H$ is the scale height. 
does not present thus behaviour. Although the $\mathrm{H} \alpha$ signal is clear and shows stability through bootstrap analysis, the origin of this feature in the transmission spectrum is not completely clear.

The exoplanet HD 209458b is highly irradiated and atmospheric conditions allow $\mathrm{Ca}$ to be in the gaseous state given observed conditions in Vidal-Madjar et al. (2011b,a) and our observed altitudes. According to the Clausius-Clapeyron analysis, Sc could condensate forming clouds or hazes.

We note that even if models predict the weakening and vanishing of $\mathrm{Ca}, \mathrm{TiO}, \mathrm{VO}$, and $\mathrm{O}$, for example via $\mathrm{CaTiO}_{3}$ (see Sect. 3.3 in Jensen et al. 2012; Allard \& Homeier 2012), the atmosphere of HD 209458b presents O (Vidal-Madjar et al. 2004), possibly TiO (Désert et al. 2008), and here we present Ca detection.

We demonstrated the potential of an automatic and blind search for evidence of exoplanetary atmospheres in the transmission spectrum resulting in the detection of species that, at the present time, have been not predicted by theoretical models. Additionally, Fossati et al. (2010) detected metals in the atmosphere of WASP-12b, Sc among them. These results show that HD 209458b and WASP-12b are both highly irradiated hotJupiters and they may present a similar photochemistry.

We also validate a method to perform telluric correction using science data itself, without the need of a reference star or models. We realize that the validity of this method is limited to data acquired in one stable single night as it involves a minimum of variation in the contents of the Earth's atmospheric components.

Acknowledgements. N.A.-D. acknowledges support from GEMINI-CONICYT FUND and from Programa Nacional de Becas de Posgrado (Grant D-22111791). P.R. and N.A.-D. acknowledge partial support from FONDECYT \#11080271 and \#1120299. The authors gratefully acknowledge the valuable comments of the anonymous referee.

\section{References}

Allard, F., Homeier, D., Freytag, B., \& Sharp, C. M. 2012, EAS Publ. Ser., 57, 3

Baba, H., Yasuda, N., Ichikawa, S.-I., et al. 2002, in ADASS XI, eds. D. A. Bohlender, D. Durand, \& T. H. Handley, ASP Conf. Ser., 281, 298

Bean, J. L., Miller-Ricci Kempton, E., \& Homeier, D. 2010, Nature, 468 , 669

Beaulieu, J. P., Kipping, D. M., Batista, V., et al. 2010, MNRAS, 409, 963

Burrows, A., Rauscher, E., Spiegel, D. S., \& Menou, K. 2010, ApJ, 719, 341

Charbonneau, D., Brown, T. M., Noyes, R. W., \& Gilliland, R. L. 2002, ApJ, 568,377

Désert, J.-M., Vidal-Madjar, A., Lecavelier Des Etangs, A., et al. 2008, A\&A, 492,585

Fortney, J. J., Shabram, M., Showman, A. P., et al. 2010, ApJ, 709, 1396

Fossati, L., Haswell, C. A., Froning, C. S., et al. 2010, ApJ, 714, L222

Jensen, A. G., Redfield, S., Endl, M., et al. 2011, ApJ, 743, 203

Jensen, A. G., Redfield, S., Endl, M., et al. 2012, ApJ, 751, 86

Knutson, H. A., Charbonneau, D., Noyes, R. W., Brown, T. M., \& Gilliland, R. L. 2007, ApJ, 655, 564

Langland-Shula, L. E., Vogt, S. S., Charbonneau, D., Butler, P., \& Marcy, G. 2009, ApJ, 696, 1355

Lide, D. R. 2010, CRC Handbook of chemistry and physics, 90th edn. (CD-ROM Version 2010) (Boca Raton, FL: CRC Press/Taylor and Francis)

Lobel, A. 2008, J. Phys. Conf. Ser., 130, 012015

Mayor, M., \& Queloz, D. 1995, Nature, 378, 355

Narita, N., Suto, Y., Winn, J. N., et al. 2005, PASJ, 57, 471

Redfield, S., Endl, M., Cochran, W. D., \& Koesterke, L. 2008, ApJ, 673, L87

Snellen, I. A. G., Albrecht, S., de Mooij, E. J. W., \& Le Poole, R. S. 2008, A\&A 487, 357

Southworth, J. 2010, MNRAS, 408, 1689

Vidal-Madjar, A., Lecavelier des Etangs, A., Désert, J.-M., et al. 2003, Nature, 422, 143

Vidal-Madjar, A., Désert, J.-M., Lecavelier des Etangs, A., et al. 2004, ApJ, 604, L69

Vidal-Madjar, A., Huitson, C. M., Lecavelier des Etangs, A., et al. 2011a, A\&A, 533, C4

Vidal-Madjar, A., Sing, D. K., Lecavelier Des Etangs, A., et al. 2011b, A\&A, 527, A110

Winn, J. N., Suto, Y., Turner, E. L., et al. 2004, PASJ, 56, 655 